\title{
Detection and Tracking of Driver's Hands in Real Time
}

\author{
Raúl Crespo, Isaac Martín de Diego, Cristina Conde, and Enrique Cabello \\ Face Recognition and Artificial Vision Group, Universidad Rey Juan Carlos. \\ C. Tulipan, S/N, 28934, Mostoles, España \\ \{raul.crespo.soto, isaac.martin, cristina.conde, \\ enrique.cabello\}@urjc.es
}

\begin{abstract}
In this paper a complete driver's hands detection and tracking system suitable for working in real time conditions has been developed. The proposed system has been successfully tested in close-real world conditions in different scenarios on a very realistic and immersive cabin truck simulator. A database of 24 video sequences monitoring the driving task in different circuits, illumination conditions and video resolutions has been obtained. The hands detection rate and the computational times needed to process each frame are presented. The proposed system has proven to be high accurate and fast enough to work in real time conditions. In the future, the selected algorithm will be included as part of an automotive compliance embedded system placed in a real truck cabin.
\end{abstract}

Keywords: Image Processing, Real Time, Tracking, Automotive Application.

\section{Introduction}

Driver's distraction is involved in $30 \%$ of the car accidents and is responsible of a lot of fatalities every year [1]. To study driver's distractions is a very difficult problem due to the high number of factors involved in the distraction-related accidents [2]. One of the most important of these parameters is the driver behavior that could be study analyzing the position of his/her hands.

There are three elements that compose an accident: vehicle, road, and driver. Among these three elements, human factor is the one that has received least attention in the past. Vehicle manufacturers have increased security measures, both active and passive. Roads have improved its quality: received new layers of asphalt, better signaling, together with more appropriate driving design. Several approaches to supervise the driving task using computer vision techniques have been presented during the last years. A driver hand supervising system based on artificial vision techniques has been presented in [3]. The hands were detected by fitting a geometrical model to them and tracking was done based on an extended Kalman Filter. Although graphical results were shown in some videos, no numerical data were presented. In addition the tests were obtained with a parked car with changes in lighting. In [4], a vision system has been proposed for the analysis of the driver's behavior based on 3D tracking of the driver's head and hands. In this case, several cameras were used. First, basic information regarding hands position was retrieved, whether the hands were placed on the wheel or not, and where the driver's gaze is set: left, right, or forward. This basic 
information was put together to generate a high level response. In order to determine the position of the hands, a semi supervised system was used. This system removed the background from color images and has been completely described in [5]. A method for head tracking has been developed in [6]. The main idea was to combine a static model with a $3 \mathrm{D}$ estimation in real time based on a tracking system. Various graphic results were presented, showing that the tests were undergone outdoors. Although it was indicated that the algorithm performs in real time, hand detection tests only reached six frames per second (fps). The objective of this the present work is to develop a hand driver supervision system with a low computational cost that allows the system to work in real time conditions, and to test it in realistic conditions. This supervision is done with a non-intrusive technical setup, using an infrared lighting system (invisible to the human eye) and a charge-coupled device (CCD) camera. The output of this system will be a signal indicating if the hands are placed on the steering wheel or in other areas of interest. Most of the alternative hands detection methods are based on the skin detection using color information. However, in our problem the color information is lost due to the special light conditions of our framework.

The rest of the paper is organized as follows: in Section 2, we describe the technical setup and the database acquisition. Section 3 presents the proposed algorithms to hand detection and tracking. The results on several videos sequences obtained from a cabin truck simulator are described in Section 4. Finally, Section 5 summarizes the conclusions and future work.

\section{Technical Setup}

CABINTEC ("Intelligent cabin truck for road transport") is an ongoing project focused on risk reduction for traffic safety [7]. One of the main objectives of CABINTEC is to identify driver's unsuitable behavior and lacks of attention. The data base considered in this paper was obtained in a very realistic and immersive cabin truck simulator (see [8] for a complete description). The cabin is a real cabin truck placed over pneumatically devices, so real movements are reproduced. The frontal and lateral views are covered by scene projectors synchronized with the driving activities. The rear mirrors are computer screens showing the rear objects. Sound simulation helps to be focused on driving actions, recreating a natural driving situation. The acquisition setup is shown in Figure 1. The whole system is very compact and is placed in the top of the truck cabin. Although in the experimental configuration shown all elements are visible, in the final configuration all elements were covered. The proposed system consists of three infrared illuminators and a CCD camera. One of the illuminators is focused on the left hand of the driver and the other two are placed up of the steering wheel with the CCD camera between them. Each of these two illuminators has a white diffuser in front of it. Illuminators emit a continuous beam of infrared light ( 850 $\mathrm{nm}$ wavelength), so it is not visible by the human eye. CCD camera captures only in its infrared-acquisition peak, using a filter that removes visible information and allows only the pass of infrared wavelength. CCD camera is a five megapixels resolution complementary metal-oxide-semiconductor (CMOS) sensor and has a maximum 


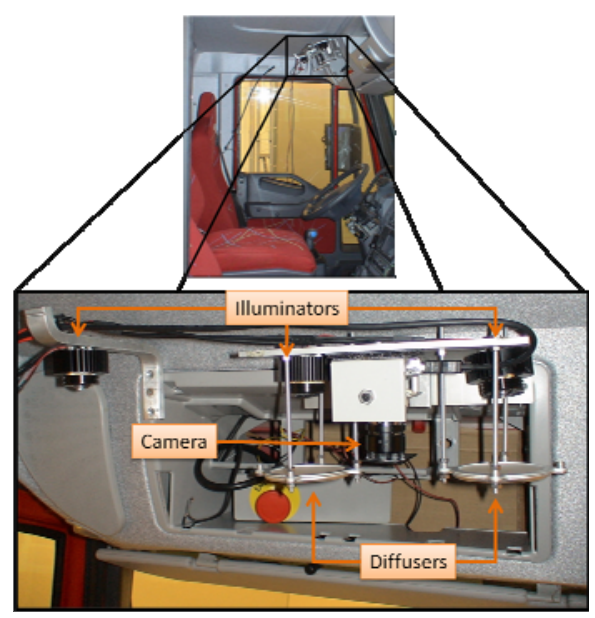

Fig. 1. Experimental setup of the camera, the illuminators and diffusers

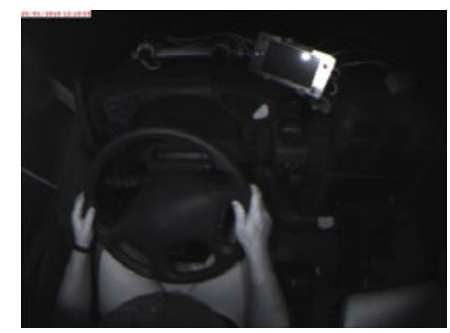

Fig. 2. Sample image taken from camera

bandwidth of 14 frames per second at full resolution. Resolution of the camera can be selected between several standard formats, in our case 352x288, 640x480 and $1024 \times 768$ are allowed modes.

Figure 2 shows a sample image acquired by the camera. Camera height is $80 \mathrm{~cm}$ on the driving wheel but its field of view includes the complete steering wheel, gear change and hand brake levers, GPS, instrument panel and part of the copilot seat.

\subsection{Data Base Description}

In order to collect the database, an experimental design considering four factors was built: driving scenario, number of illuminators, number of diffusers, and resolution sizes. Two driving scenarios were selected in the simulator: an urban path and a road between two cities (interurban path). Following the indications of the professional driver, no gloves were considered during the experiment. Hands motion while driving strongly depends on the kind of scenario, so the system response was deeply analyze in each case. Half of the sequences come from each scenario. Four illumination conditions were tested: three illuminators with two diffusers (3I2D), three illuminators with no diffusers (3I0D), two illuminators with two diffusers (2I2D) and two illuminators with no diffusers (2I0D). For each illumination condition, three resolution sizes were

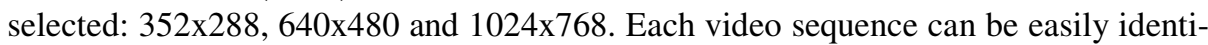
fied knowing the scenario (urban-interurban); illumination conditions (3I2D, 3I0D,

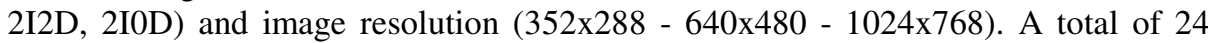
video sequences were obtained. The time length of each sequence was at least seven minutes. The average number of fps acquired is 25.6 for $352 \times 288$ image size, 12.4 for $640 \times 480$ and 5.7 for $1024 \times 768$.

To obtain the "ground truth", that is, the real position of the driver's hands, all images were manually labeled by two independent experts. The number of driver hands in each region was stored. When contradictory labels were obtain (for example, the 
first expert determines two hands on the steering wheel area, but the second expert determines only one hand), the opinion of a third expert was considered.

\section{Detection and Tracking Methods}

Three algorithms to detect the driver's hands have been developed. The first algorithm, Global Threshold Detection (GDT), makes a sequential search in the regions of interest (ROI) previously defined. It has been considered that the most important ROI is the steering wheel region. The most efforts (such as fit of relevant parameters) are focused on this region. The main difference between GDT and the second proposed algorithm, Local Threshold Detection (LTD), is the information taking into account to binaries the steering wheel area. In the GDT method a global adaptive threshold that considers the whole image is done. In the LTD method the steering wheel area has been divided into four quadrants, and a different local threshold is considered for each quadrant. Finally, in the third proposed algorithm, Local Threshold Detection with Tracking (LTD-T), a tracking module has been added to the LTD method.

\subsection{Global Threshold Detection (GDT)}

This algorithm performs a search of the hands on the steering wheel ROI, and if nohands are detected, a search on other ROIs such as gear charge and hand brake levers. First, a binarization on the steering wheel ROI is calculated. Next, a logical "and" operator is applied to the mask of the steering area and the binarized image. As a result, the occlusions on the steering area are obtained. Notice that the number of these occlusions is, in general, higher than two (the desirable number). Next, for each occlusion, we search the associated area in the binarized image. These areas are called candidates. Usually, this step eliminates errors such as shine from the steering wheel. To calculate the final points, called endpoints, several methods of discrimination between candidates are used. The candidates with size lower than a threshold were eliminated. If the distance between adjacent candidates were lower than a threshold, the candidates were merged in a unique candidate. If only one candidate remains, it is considered an endpoint, that is, a hand has been detected. If two candidates remain, it is necessary to determinate whether they correspond to one or two hands. To do that, the centers of the ellipses containing each candidate are obtained. The ellipse which major axis is the distance between these centers is built. If the percentage of the area inside the new ellipse corresponding to candidates is higher than a threshold, it means that the two candidates correspond to the same hand. For instance, this is the case of a hand and an arm "separated" for a watch. If the percentage of the area inside the new ellipse corresponding to candidates is lower than the threshold, it means that the two candidates correspond to different hands. This is the case of two hands placed in separated steering wheel areas. In the particular case when two hands were not detected, a search in other ROIs was carried out: the gear lever and the truck hand brake were the considered ROIs. To develop such searches we calculate the difference between the image in the ROI and the background image calculated when the hands were on the steering wheel. An illustrative example of the GDT algorithm is presented in Figure 3. 


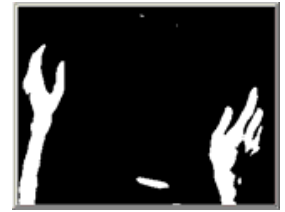

(a) Adaptive

Threshold result.

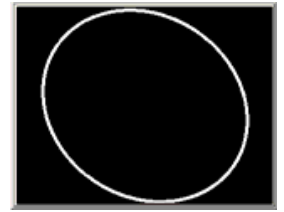

(b) Mask of the steering wheel area.

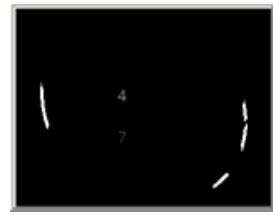

(c) Occlusions on the steering wheel.

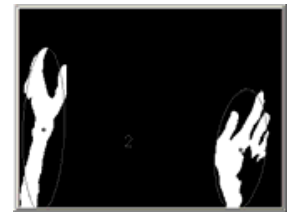

(d) Endpoints.

Fig. 3. Steps of the GDT algorithm for a sample image

\subsection{Local Threshold Detection (LTD)}

In this algorithm a new method to binarize the steering wheel area is proposed. In this case local thresholds are considered for each part of the wheel, making the system more adaptive to changing illumination. The ROI of the steering wheel is divided into four quadrants as shown in Figure 4(a). Different thresholds are calculated for each quadrant. Then, each of these quadrants is locally binarized with their local thresholds. As experiments will show in Section 4, this change reduces the processing time of the algorithm without worsening the GDT detection results. Furthermore, the LTD method obtains candidates with more smooth edges than those obtained when GDT algorithm was used (see Figure 3(d) and Figure 4(c), as example).

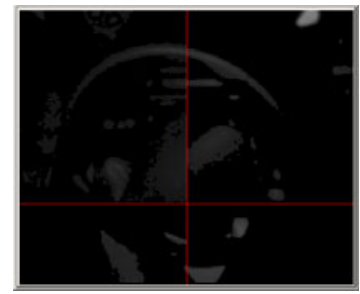

(a) ROI divided in four quadrants.

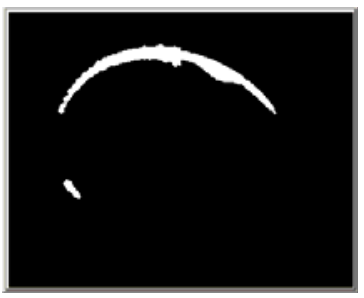

(b) Initially the segmentation fail because the threshold levels are unknown.

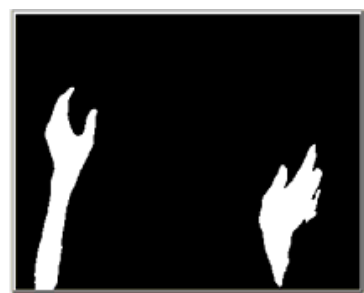

(c) Binarization with the correct threshold.

Fig. 4. Steps of the LDT algorithm for a sample image

\subsection{Local Threshold Detection with Tracking (LTD-T)}

In order to improve the LTD algorithm, a simple and soft tracking module based on Camshift algorithm [9] has been integrated. As a first step of the LTD-T algorithm, we detect the hands with the LTD method previously described. Once we know the hand positions, the well known Camshift algorithm is used for the tracking step. If hands are lost during the tracking step, the LTD method is used to detect them again. In order to define the model of the object to track, the Camshift algorithm needs a segmented image as parameter. To obtain this image the difference between the actual frame and the image mode is used. To control the performance of the algorithm, the detection step is executed even when the hands are not missed in the tracking step. This control is developed each 20 frames, that is, approximately once per second. 


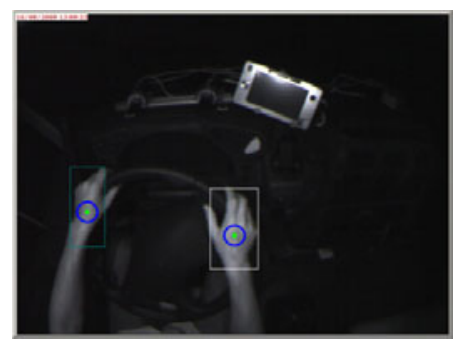

Fig. 5. The tracking windows of LTD-T algorithm

Given the robustness of the LTD algorithm, if the detection step and the tracking step locate the hands in different positions, only the detection step location is considered.

\section{Experiments and Results}

The performance of the three proposed algorithms was analyzed in the close-real world conditions of the 24 video sequences described in Section 2. The hand detection results are presented in Table 1. For each frame of the videos, the ground truth defined in Section 2 was compared with the detection results (number of hands in the ROI) of each algorithm for that frame.

Table 1. Hands detection results, percentage of success, for GDT, LTD and LTD-T algorithms in all the proposed configurations (2I0D: 2 illuminators and 0 diffusers, 2I2D: 2 illuminators and 2 diffusers, 3IOD: 3 illuminators and 0 diffusers, 3I2D: 3 illuminators 2 diffusers)

\begin{tabular}{|c|c|c|c|c|c|c|c|c|c|}
\hline & & \multicolumn{4}{|c|}{ Interurban } & \multicolumn{4}{|c|}{ Urban } \\
\hline & & 2I0D & $2 \mathrm{I} 2 \mathrm{D}$ & 3I0D & $3 \mathrm{I} 2 \mathrm{D}$ & 2I0D & $2 \mathrm{I} 2 \mathrm{D}$ & 3I0D & $3 \mathrm{I} 2 \mathrm{D}$ \\
\hline \multicolumn{10}{|l|}{ GDT } \\
\hline & $352 \times 288$ & 7 & 13 & 90 & 87 & 43 & 23 & 83 & 84 \\
\hline & $640 \times 480$ & 75 & 12 & 98 & 93 & 69 & 81 & 86 & 88 \\
\hline & $1024 \times 768$ & 92 & 19 & 93 & 90 & 71 & 43 & 90 & 85 \\
\hline \multicolumn{10}{|l|}{ LTD } \\
\hline & $352 \times 288$ & 88 & 70 & 91 & 90 & 70 & 58 & 83 & 82 \\
\hline & $640 \times 480$ & 96 & 96 & 98 & 93 & 68 & 83 & 81 & 88 \\
\hline & $1024 \times 768$ & 92 & 76 & 77 & 61 & 56 & 42 & 81 & 52 \\
\hline \multicolumn{10}{|l|}{ LTD-T } \\
\hline & $352 \times 288$ & 79 & 71 & 91 & 94 & 61 & 64 & 83 & 83 \\
\hline & $640 \times 480$ & 96 & 97 & 94 & 91 & 75 & 76 & 84 & 85 \\
\hline & $1024 \times 768$ & 89 & 83 & 88 & 86 & 76 & 43 & 89 & 94 \\
\hline
\end{tabular}

In general, 3 illuminators schemes outperform 2 illuminators schemes. No significative differences were observed between 0 and 2 diffusers. In the interurban circuit OD configuration seems to be better, but in the urban circuit $2 \mathrm{D}$ configuration seems to outperform 0D. As expected, the results in the interurban circuit are slightly better than the results in the urban circuit. The driver moved his hands most frequently in 
the urban than in the interurban scenario in order to response to the circuit design: curves, traffic signs, etc. Regarding image size, the best results were obtained for the $640 x 480$ configuration. As a global conclusion the best configuration corresponds to: 3I0D and 640x480 image size. For the GDT method the best overall detection result for interurban circuit was achieved for 3I0D and 640x480 image size configuration (98\%). For the urban circuit case, the best accuracy was achieved for 3I0D and $1024 \times 762$ image size (90\%). As in the GDT algorithm, for the LTD method the best detection result for interurban circuit was achieved for 3I0D and 640x480 image size configuration (98\%). For the urban circuit case, the best result was achieved for 3I2D and 640x480 image size (88\%). For the LTD-T method the best result for interurban circuit was achieved for 2I2D and 640x480 image size configuration (97\%). For the urban circuit case, the best result was achieved for 3I2D and $1024 \times 762$ image size (94\%). Notice that increasing the image resolution the performance is not generally improved.

As a resume, the LTD-T method obtains better results than GDT method in 14 of 24 configurations. In addition the LTD-T method is better than LTD method in 16 of 24 sequences. If we considered as significative difference between detection percentages those higher than 5\%, LTD-T algorithm outperforms GDT algorithm in 9 configurations. GDT outperforms LTD-T only in 1 configuration. In 14 configurations no significative differences were observed. Using the same definition of significative difference, the LTD-T method outperforms LTD method in 8 configurations. LTD outperforms LTD-T only in 3 configurations. In 13 configurations no significative differences were observed. That is, LTD-T algorithm improves GDT and LTD algorithms in most of the configurations analyzed.

The average computational times needed to process each frame in a PC (CORE 2 DUO $3.00 \mathrm{GHz}$ ) are presented in Table 2. For all the image sizes, the faster algorithm is LTD, and the slowest algorithm is GTD. As expected, the LTD-T algorithm is slightly slower than the LTD algorithm. The main reason of this difference is that when the LTD-T algorithm misses some of the hands during the tracking step, an additional detection step is needed to recover the hands. That is, for the same frame, two procedures are performed.

Table 2. Average time in milliseconds needed to process each frame

\begin{tabular}{lccc}
\hline & $352 \times 288$ & $640 \times 480$ & $1024 \times 768$ \\
\hline GDT & 12 & 23 & 102 \\
LTD & 3 & 7 & 18 \\
LTD-T & 8 & 17 & 41 \\
\hline
\end{tabular}

\section{Conclusions and Future Work}

In this paper, a complete system of driver's hands detection and tracking is presented. Three different algorithms have been developed and tested in a large database acquired in a high realistic truck simulator, close to real world conditions. The performance of each algorithm has been tested in different illumination conditions and image resolutions. The LTD-T algorithm gives the most complete information: not only 
allows the detection of the hands on the ROIs but the position of the hands on the complete image is known in each frame. The algorithm can automatically detect and track the hands in real time. Regarding the detection task, the LTD-T improves the GDT and the LTD methods. With the appropriate illumination and resolution, the accurate of the system is higher than $90 \%$. Most of the errors were due to hands passing over each other during heavy turns of the steering wheel. As future work, the LTD-T algorithm will be included as part of an automotive compliance embedded system to be set in a real truck cabin. An alert will be given when the LTD-T algorithm detects a lack of attention regarding the hands position. In addition, the system will consider other signals such as head position in face images.

\section{Acknowledgements}

Supported by the Minister for Science and Innovation of Spain through the projects CABINTEC: PSE-37010-2007-2 and VULCANO: TEC2009-10639-C04-04.

\section{References}

1. Dingus, T., et al.: The 100-Car Naturalistic Driving Study. Virginia Tech Transportation Institute, NHTS (2006)

2. Zhang, H., Schreiner, C., Zhang, K., Torkkola, K.: Naturalistic use of cell phones in driving and context-based user assistance. In: Lew, M., Sebe, N., Huang, T.S., Bakker, E.M. (eds.) HCI 2007. LNCS, vol. 4796, pp. 273-276. Springer, Heidelberg (2007)

3. McAllister, G., McKenna, S.J., Ricketta, I.W.: Tracking a driver's hands using computer vision. In: IEEE International Conference on Systems, Man, and Cybernetics (2000)

4. Tran, C., Trivedi, M.M.: Driver Assistance for "Keeping Hands on the Wheel and Eyes on the Road". In: IEEE International Conference on Vehicular Electronics and Safety (2009)

5. Tran, C., Trivedi, M.M.: Introducing XMOB: Extremity Movement Observation Framework for Upper Body Pose Tracking in 3D. In: IEEE Int. Symp. on Multimedia (2009)

6. Murphy-Chutorian, E., Trivedi, M.M.: HyHOPE: Hybrid Head Orientation and Position Estimation for Vision-based Driver Head Tracking. In: IEEE Int. Vehicles Symposium (2008)

7. Brazalez, A., Delgado, B., Sevillano, M., Garcia, I., Matey, L.: Cabintec: Cabina inteligente para el transporte por carretera. In: Proc. VIII Cong. Esp. Sist. Int. de Transporte (2008)

8. Siordia, O.S., Martín de Diego, I., Conde, C., Reyes, G., Cabello, E.: Driving risk in, classification based on experts evaluation. In: Proceedings of the 2010 IEEE Intelligent Vehicles Symposium, IV 2010 (2010)

9. Bradski, G.R.: Computer vision face tracking for use in a perceptual user interface. Intel Technology Journal, 2nd Quarter (1998) 